\title{
The evolutionary state of the southern dense core Chamaeleon-MMS1
}

\author{
A. Belloche ${ }^{1}$, B. Parise ${ }^{1}$, F. F. S. van der Tak ${ }^{1, \star}$, P. Schilke ${ }^{1}$, S. Leurini ${ }^{1}$, R. Güsten ${ }^{1}$, and L.- $\AA$. Nyman ${ }^{2}$ \\ 1 Max-Planck-Institut für Radioastronomie, Auf dem Hügel 69, 53121 Bonn, Germany \\ e-mail: belloche@mpifr-bonn.mpg.de \\ 2 European Southern Observatory, Alonso de Cordova 3107, Casilla 19001, Santiago 19, Chile
}

Received 29 March 2006; Accepted 18 May 2006

ABSTRACT

\begin{abstract}
Aims. Our goal is to set constraints on the evolutionary state of the dense core Cha-MMS1 in the Chamaeleon I molecular cloud. Methods. We analyze molecular line observations carried out with the new submillimeter telescope APEX. We look for outflow signatures around the dense core and probe its chemical structure, which we compare to predictions of models of gas-phase chemistry. We also use the public database of the Spitzer Space Telescope (SST) to compare Cha-MMS1 with the two Class 0 protostars IRAM 04191 and L1521F, which are at the same distance.

Results. We measure a large deuterium fractionation for $\mathrm{N}_{2} \mathrm{H}^{+}(11 \pm 3 \%)$, intermediate between the prestellar core L1544 and the very young Class 0 protostar L1521F. It is larger than for $\mathrm{HCO}^{+}(2.5 \pm 0.9 \%)$, which is probably the result of depletion removing $\mathrm{HCO}^{+}$from the high-density inner region. Our $\mathrm{CO}(3-2)$ map reveals the presence of a bipolar outflow driven by the Class I protostar Ced 110 IRS 4 but we do not find evidence for an outflow powered by Cha-MMS1. We also report the detection of Cha-MMS1 at 24, 70 and $160 \mu \mathrm{m}$ by the instrument MIPS of the SST, at a level nearly an order of magnitude lower than IRAM 04191 and L1521F. Conclusions. Cha-MMS1 appears to have already formed a compact object, either the first hydrostatic core at the very end of the prestellar phase, or an extremely young protostar that has not yet powered any outflow, at the very beginning of the Class 0 accretion phase.
\end{abstract}

Key words. stars: formation - ISM: individual objects: Cha-MMS1 - ISM: abundances - astrochemistry - ISM: jets and outflows

\section{Introduction}

The study of the earliest phases of star formation, the so-called prestellar and Class 0 stages, is essential to understand the origin of the stellar initial mass function (e.g. Ward-Thompson et al. 2006). As one of the closest active sites of low-mass star formation, the Chamaeleon I dark cloud is an excellent target to make progress in this field. However, its population of prestellar cores is not known and only 1 or 2 Class 0 protostellar candidates have been reported so far (see Reipurth et al. 1996; Froebrich 2005). The most promising of them, Cha-MMS1, is embedded in a $\mathrm{C}^{18} \mathrm{O}(1-0)$ clump (Haikala et al. 2005), in the Cederblad 110 region where several young stellar objects have been identified in the infrared (e.g. Prusti et al. 1991; Persi et al. 2001). Using a tentative far-infrared detection, Lehtinen et al. (2001) derived a low temperature $\left(T_{\text {bol }}=20 \mathrm{~K}\right)$ and luminosity $\left(L_{\mathrm{bol}}=0.45 L_{\odot}\right)$ for Cha-MMS1. They proposed that it is a Class 0 protostar, in agreement with Reipurth et al. (1996) who suggested that it could be the driving source of the Herbig-Haro objects and the CO outflow seen nearby. However Lehtinen et al. (2003) failed to detect $\mathrm{cm}$-wave emission with the ATCA interferometer, suggesting it is still prestellar. Here, in an effort to clarify its evolutionary state, we report molecular line observations carried out with the new submillimeter telescope $\mathrm{APEX}^{1}$ and the infrared

\footnotetext{
* Present address: SRON, 9747 AD Groningen, The Netherlands

1 This publication is based on data acquired with the Atacama Pathfinder Experiment (APEX). APEX is a collaboration between the Max-Planck-Institut für Radioastronomie, the European Southern Observatory, and the Onsala Space Observatory.
}

detection of Cha-MMS1 at 24, 70 and $160 \mu \mathrm{m}$ by the Spitzer Space Telescope.

\section{Observations}

We observed the dense core Cha-MMS1 $\left(\alpha_{2000}=11^{\mathrm{h}} 06^{\mathrm{m}} 31^{\mathrm{s}} \mathrm{7}\right.$, $\delta_{2000}=-77^{\circ} 23^{\prime} 33^{\prime \prime}$, Reipurth et al. 1996) in July, September and November 2005 with the APEX telescope (Güsten et al., this volume). The double-side-band heterodyne receiver APEX2A (Risacher et al., this volume) was tuned to the molecular transitions listed in Table 2. The $\mathrm{N}_{2} \mathrm{H}^{+}(4-3)$ line was observed simultaneously with the $\mathrm{H}_{2} \mathrm{D}^{+}$line in the $1 \mathrm{GHz}$ bandpass. The half-power beamwidth was $18^{\prime \prime}$ at $345 \mathrm{GHz}$, and the forward and beam efficiencies used to convert antenna temperatures $T_{\mathrm{a}}^{\star}$ into main-beam temperatures $T_{\mathrm{mb}}$ were 0.97 and 0.73 , respectively. The double-side-band system temperatures (continuum calibration) ranged from 100 to $360 \mathrm{~K}$ in $T_{\mathrm{a}}^{\star}$ scale. From spectra taken on different days, we estimate the calibration uncertainty better than $\sim 15 \%$. The backend was a Fast-Fourier-Transform spectrometer with 16384 channels and a channel spacing of $61 \mathrm{kHz}$ yielding an effective spectral resolution of about $120 \mathrm{kHz}$ (Klein et al., this volume). The telescope pointing was checked on RDor, 07454-7112, IRAS 15194-5115, or Mars and found to be accurate to $\sim 4^{\prime \prime}$ (rms). The telescope focus was optimized on Saturn or Mars. The observations were performed in positionswitching mode using the APECS software (Muders et al., this volume). The data were reduced with the CLASS software (see http://www.iram. fr/IRAMFR/GILDAS). 

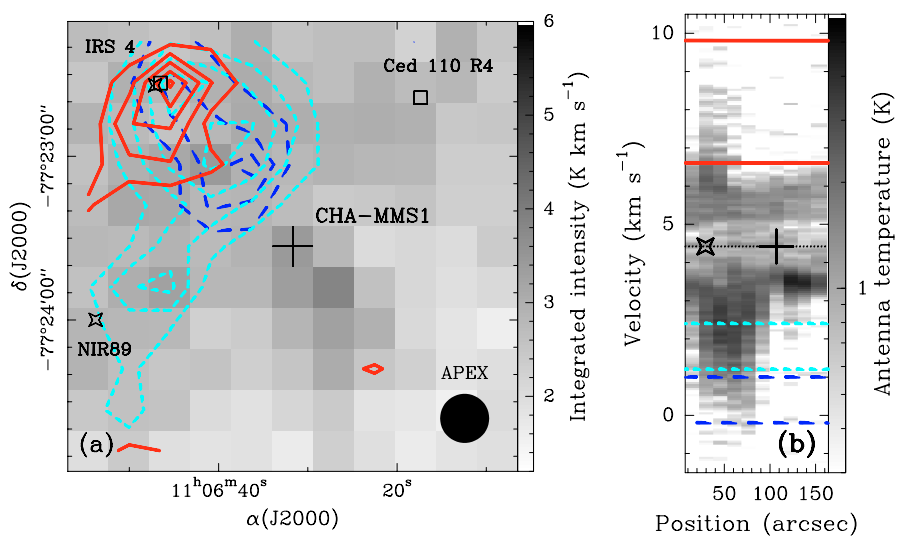

Fig. 1. a) $\mathrm{CO}(3-2)$ integrated intensity observed with APEX toward Cha-MMS1 (cross). The emission integrated over $[3.6,5.8] \mathrm{km} \mathrm{s}^{-1}$ is shown in greyscale. The blueshifted, less blueshifted and redshifted emissions integrated over $[-0.2,1.0],[1.2,2.4]$ and $[6.6,9.8] \mathrm{km} \mathrm{s}^{-1}$ are plotted as long-dashed, short-dashed and thick contours, respectively. The steps are $3 \sigma, 6 \sigma$ and $3 \sigma$ with rms $\sigma=0.13,0.13$ and 0.24 $\mathrm{K} \mathrm{km} \mathrm{s}^{-1}$, respectively. The HPBW is shown on the right. The star and square symbols mark the position of near-infrared (Persi et al. 2001) and $3.5 \mathrm{~cm}$ sources (Lehtinen et al. 2003). b) $\mathrm{CO}(3-2)$ position-velocity diagram along the axis going through IRS 4 and Cha-MMS1. The dotted line indicates the systemic velocity of Cha-MMS1. The other lines show the limits of the velocity ranges used for the contour maps. The logarithmic temperature scale varies from 0.3 to $6 \mathrm{~K}$.

\section{Analysis: kinematics and deuteration}

\subsection{No evidence for an outflow driven by Cha-MMS1}

We mapped the region around Cha-MMS1 in $\mathrm{CO}(3-2)$ with a spacing of $15^{\prime \prime}$. The spectra are deeply self-absorbed. They are very broad with stronger wing emission in the northeastern part. Figure 1a shows maps of the emission integrated over the core and the wings of the line. The blueshifted and redshifted emissions are clearly associated with the Class I protostar Ced 110 IRS 4 and very likely trace a bipolar outflow. Their morphology and the position-velocity diagram shown in Fig. 1b fall into case 1 of Cabrit \& Bertout (1990), which suggests that the outflow axis is close to the line of sight $\left(i \lesssim 30^{\circ}\right)$. The outflow geometry is consistent with the geometry of the bipolar nebula seen in the near infrared around IRS 4 (Zinnecker et al. 1999). The emission integrated over less blueshifted velocities traces the same lobe close to IRS 4 but in addition shows a prominent extension toward the near-infrared source NIR89, a potential Class I young brown dwarf (Persi et al. 2001). This additional wing emission could be associated with another outflow driven by NIR89, since Prusti et al. (1991) detected an elongated redshifted emission in $\mathrm{CO}(1-0)$ on the other side of NIR89 (see their Fig. 5). This potential second outflow would have an intermediate inclination $\left(20^{\circ} \lesssim i \lesssim 70^{\circ}\right)$ since its two lobes do not overlap. On the other hand, we do not find significant wing emission in the vicinity of Cha-MMS1, which indicates that it does not drive an outflow on the scale probed with APEX.

\subsection{Physical structure of Cha-MMS1}

We derive the density structure of Cha-MMS1 from $1.3 \mathrm{~mm}$ continuum measurements done with SEST (Reipurth et al. 1996). Assuming a uniform dust temperature of $10 \mathrm{~K}$, optically thin emission and a dust opacity $\kappa_{1.3 \mathrm{~mm}}=0.01 \mathrm{~cm}^{2} \mathrm{~g}^{-1}$ (van der Tak et al. 1999), we derive a mass of $0.40 M_{\odot}$ in the $22^{\prime \prime}$ SEST beam and $1.0 M_{\odot}$ in the region within the $5 \sigma$ contour level of

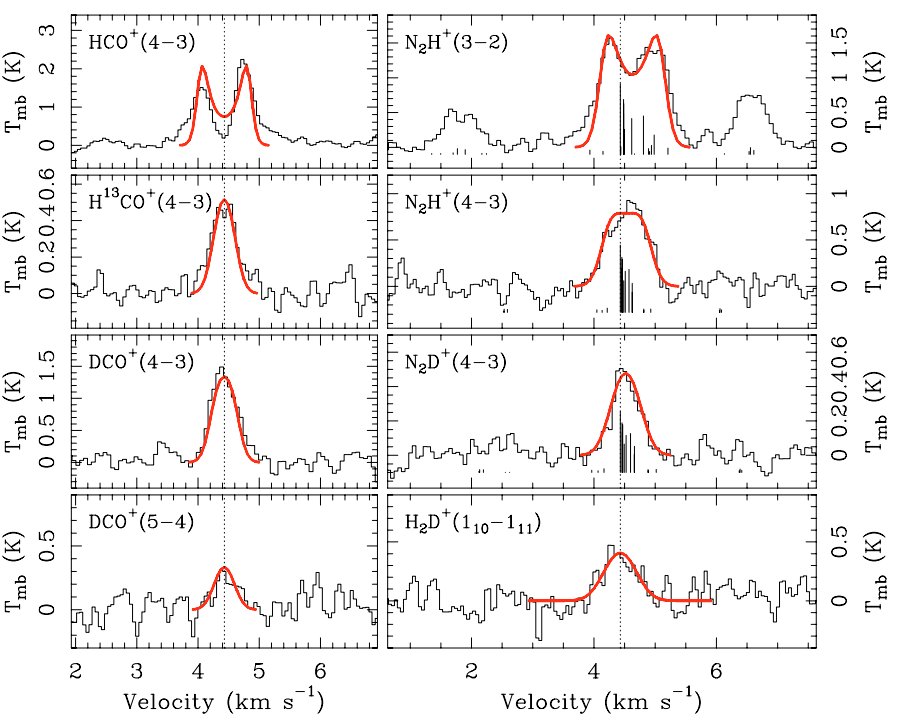

Fig. 2. Spectra observed with APEX toward Cha-MMS1 (histogram). Synthetic spectra corresponding to the model of Sect. 3.3 are superimposed (thick line). The hyperfine structure of the $\mathrm{N}_{2} \mathrm{H}^{+}$and $\mathrm{N}_{2} \mathrm{D}^{+}$lines is shown under each spectrum with statistical weighting. The dotted line indicates the systemic velocity assumed for the modeling.

Reipurth et al. (1996), which has a deconvolved diameter of $\sim 32^{\prime \prime}$. If the dense core has a flat inner region and a density decreasing as $r^{-2}$, which is typical for prestellar condensations (e.g. Bacmann et al. 2000), then the two measurements yield a central density $n_{\mathrm{H}_{2}, \mathrm{c}}=3.3_{-0.6}^{+0.4} \times 10^{6} \mathrm{~cm}^{-3}$ and a radius of the flat inner region $r_{\text {flat }}=1800_{-200}^{+450} \mathrm{AU}$ at a distance of $150 \mathrm{pc}$ (Knude \& Høg 1998).

\subsection{Molecular abundances and deuteration}

The spectra obtained with APEX toward the continuum peak position of Cha-MMS1 are presented in Fig. 2 and the results of single Gaussian and 7-component HFS fits done with the CLASS software are written in Table 1 . The systemic velocities derived from the fits agree quite well except for $\mathrm{N}_{2} \mathrm{H}^{+}$(4-3) and $\mathrm{H}_{2} \mathrm{D}^{+}\left(1_{10}-1_{11}\right)$. Since these two lines were observed simultaneously in the same bandpass, we suspect an instrumental problem and have shifted these two spectra by $-0.20 \mathrm{MHz}$ in Fig. 2 to compensate for this difference. In the following, we assume a systemic velocity of $4.43 \mathrm{~km} \mathrm{~s}^{-1}$. The opacity of $\mathrm{N}_{2} \mathrm{D}^{+}(4-$ 3 ) is small and hence not well constrained by the HFS fit. We fixed it to 0.5 (see model below). The opacity of $\mathrm{N}_{2} \mathrm{H}^{+}(3-2)$ is also uncertain, although this is not seen in the statistical uncertainty ${ }^{2}$. Assuming a kinetic temperature of $10 \mathrm{~K}$, the non-thermal linewidths are between $\sim 0.4$ and $\sim 0.5 \mathrm{~km} \mathrm{~s}^{-1}$. The linewidths derived from the HFS fits should not be affected by optical depth effects. They agree with each other within $2 \sigma$ and we find a weighted mean $\Delta V_{\mathrm{NT}}=0.40 \pm 0.02 \mathrm{~km} \mathrm{~s}^{-1}$. This corresponds to a non-thermal dispersion $\sigma_{\mathrm{NT}}=0.17 \pm 0.01 \mathrm{~km} \mathrm{~s}^{-1}$ which is on the same order as the thermal velocity dispersion at $10 \mathrm{~K}$. There is therefore a significant amount of non-thermal motions in the dense core.

The $\mathrm{HCO}^{+}(4-3)$ spectrum is strongly self-absorbed and asymmetric, the red peak being stronger than the blue one, and

\footnotetext{
2 The self-absorption of the central group of hyperfine components lowers its "mean peak temperature" and the opacity derived by the HFS fit is then artificially increased. For test purposes, we masked out the self-absorption and the opacity was reduced to $19.6 \pm 1.1$.
} 
Table 1. Results of single Gaussian (top) and 7-component HFS (bottom) fits to the spectra observed toward the center of Cha-MMS1.

\begin{tabular}{|c|c|c|c|c|c|c|}
\hline Line & $\begin{array}{c}V_{\mathrm{lsr}}^{(1)} \\
\left(\mathrm{km} \mathrm{s}^{-1}\right)\end{array}$ & $\begin{array}{c}F W H M^{(1)} \\
\left(\mathrm{km} \mathrm{s}^{-1}\right)\end{array}$ & $\begin{array}{c}\text { Area }^{(1)} \\
\left(\mathrm{K} \mathrm{km} \mathrm{s}^{-1}\right)\end{array}$ & $\begin{array}{l}T_{\mathrm{a}}^{\star} \\
(\mathrm{K})\end{array}$ & $\begin{array}{l}\mathrm{rms} \\
(\mathrm{mK})\end{array}$ & $\begin{array}{l}\Delta V_{\mathrm{NT}}^{(1,2)} \\
\left(\mathrm{km} \mathrm{s}^{-1}\right)\end{array}$ \\
\hline $\mathrm{H}^{13} \mathrm{CO}^{+}(4-3)$ & $4.42(8)$ & $0.53(3)$ & $0.20(1)$ & 0.35 & 41 & $0.53(3)$ \\
\hline $\mathrm{DCO}^{+}(4-3)$ & $4.42(1)$ & $0.51(3)$ & $0.58(3)$ & 1.08 & 90 & $0.49(3)$ \\
\hline $\mathrm{DCO}^{+}(5-4)$ & $4.47(4)$ & $0.46(8)$ & $0.095(15)$ & 0.20 & 71 & $0.44(8)$ \\
\hline $\mathrm{H}_{2} \mathrm{D}^{+}\left(1_{10}-1_{11}\right)$ & $4.57(4)$ & $0.63(7)$ & $0.20(2)$ & 0.31 & 78 & $0.53(8)$ \\
\hline$\overline{\text { Line }}$ & $\begin{array}{c}V_{\mathrm{lss}}^{(1)} \\
\left(\mathrm{km} \mathrm{s}^{-1}\right)\end{array}$ & $\begin{array}{c}F W H M^{(1)} \\
\left(\mathrm{km} \mathrm{s}^{-1}\right)\end{array}$ & $\begin{array}{c}T_{\mathrm{a}}^{\star} \times \tau_{\text {tot }}{ }^{(1)} \\
(\mathrm{K})\end{array}$ & $\tau_{\text {tot }}^{(1)}$ & $\begin{array}{l}\mathrm{rms} \\
(\mathrm{mK})\end{array}$ & $\begin{array}{l}\Delta V_{\mathrm{NT}}^{(1,2)} \\
\left(\mathrm{km} \mathrm{s}^{-1}\right)\end{array}$ \\
\hline $\mathrm{N}_{2} \mathrm{H}^{+}(3-2)$ & $4.44(1)$ & $0.42(1)$ & $24(2)$ & $24(2)$ & 50 & $0.40(1)$ \\
\hline $\mathrm{N}_{2} \mathrm{H}^{+}(4-3)$ & $4.65(2)$ & $0.50(5)$ & $2.3(7)$ & $3.4(14)$ & 81 & $0.49(5)$ \\
\hline $\mathrm{N}_{2} \mathrm{D}^{+}(4-3)$ & $4.44(2)$ & $0.41(3)$ & $0.53(3)$ & $0.5(0)$ & 44 & $0.40(3)$ \\
\hline
\end{tabular}

(1) The statistical uncertainties in parenthesis are given in units of the last digit. 0 means the parameter was fixed. For $V_{\text {lss }}$, they include the contribution of the frequency uncertainties listed in Table 2.

(2) The non-thermal linewidth was computed assuming $T_{\mathrm{K}}=10 \mathrm{~K}$ and, for the Gaussian fits, optically thin emission.

the low-optical depth $\mathrm{H}^{13} \mathrm{CO}^{+}$(4-3) line peaks in between. The asymmetry of the $\mathrm{HCO}^{+}(4-3)$ line actually changes across our small map: the blue peak is stronger than the red one in the southern part below $\left(0,-10^{\prime \prime}\right)$. This pattern could result from rotation (e.g. Redman et al. 2004) or a combination of infall and rotation, provided the actual center of the core is slightly offset from our "central" position toward the South (by $\sim 5^{\prime \prime}$ ). However we did not detect a clear overall velocity gradient in our $\mathrm{N}_{2} \mathrm{H}^{+}(4-$ 3 ) map over $50^{\prime \prime}$ that would unambiguously reveal the presence of rotation.

We used the Monte-Carlo-based radiative-transfer code MAPYSO (see Belloche et al. 2002 and references therein) to model the emission in $\mathrm{HCO}^{+}, \mathrm{H}^{13} \mathrm{CO}^{+}, \mathrm{DCO}^{+}, \mathrm{N}_{2} \mathrm{H}^{+}$and $\mathrm{N}_{2} \mathrm{D}^{+}$. For all species, we used the collision rates of $\mathrm{HCO}^{+}$with $\mathrm{H}_{2}$ (Flower 1999). We modeled the $\mathrm{N}_{2} \mathrm{H}^{+}$and $\mathrm{N}_{2} \mathrm{D}^{+}$lines as simple lines, without the hyperfine structure. The $\mathrm{H}_{2} \mathrm{D}^{+}$emission was modeled with the Monte-Carlo program RATRAN (see Hogerheijde \& van der Tak 2000). We used the density profile described in Sect. 3.2 and the velocity was set to zero. We assumed uniform gas temperature, abundance and nonthermal broadening for each molecule. We fixed the isotopic ratio $\frac{\left[\mathrm{HCO}^{+}\right]}{\left[\mathrm{H}^{13} \mathrm{CO}^{+}\right]}$to 70 .

The synthetic spectra of the best-fit model are overlaid in Fig. 2 and the parameters are shown in Table 2. The total opacities that we estimate from $\tau_{\text {peak }}$ using the statistical weights agree well with the opacities derived from the 7-component HFS fits (see Table 1 and Footnote 2). The non-thermal broadening derived for $\mathrm{HCO}^{+}$and its isotopologues is more reliable than the one measured in Table 1 because the opacity broadening is properly taken into account in the modeling. On the other hand, it is less reliable for $\mathrm{N}_{2} \mathrm{H}^{+}$and $\mathrm{N}_{2} \mathrm{D}^{+}$since part of it mimics the broadening by the hyperfine structure not implemented in our model. The non-thermal broadening derived for $\mathrm{H}_{2} \mathrm{D}^{+}$is larger than for $\mathrm{HCO}^{+}$and its isotopologues, but given the low signal-to-noise ratio of the $\mathrm{H}_{2} \mathrm{D}^{+}$spectrum, it may not be significant. The one measured for $\mathrm{N}_{2} \mathrm{H}^{+}$and $\mathrm{N}_{2} \mathrm{D}^{+}$in Table 1 lies in between. From the abundances obtained in Table 2 we derive the following deuterium fractionations: $\frac{\left[\mathrm{N}_{2} \mathrm{D}^{+}\right]}{\left[\mathrm{N}_{2} \mathrm{H}^{+}\right]}=11 \pm 3 \%$ and $\frac{\left[\mathrm{DCO}^{+}\right]}{\left[\mathrm{HCO}^{+}\right]}=2.5 \pm 0.9 \%$. The deuterium fractionation is therefore $\sim 4$ times larger for $\mathrm{N}_{2} \mathrm{H}^{+}$than for $\mathrm{HCO}^{+}$.
Table 2. Gas temperature, non-thermal broadening and abundance of each molecule for the best-fit model. The peak opacity of each modeled transition is also given, as well as an estimation of the total opacity of the hyperfine multiplets if they had been modeled.

\begin{tabular}{|c|c|c|c|c|c|c|c|}
\hline Molec. & Line & $\begin{array}{c}\text { Frequency }^{(1)} \\
(\mathrm{MHz})\end{array}$ & $\begin{array}{l}T_{\mathrm{k}} \\
(\mathrm{K})\end{array}$ & $\begin{array}{c}\Delta V_{\mathrm{NT}} \\
\left(\mathrm{km} \mathrm{s}^{-1}\right)\end{array}$ & $\chi^{(2)}$ & $\tau_{\text {peak }}$ & $\tau_{\text {tot }}$ \\
\hline $\mathrm{HCO}^{+}$ & $4-3$ & $356734.134(50)$ & 8.8 & 0.35 & $8.4(-10)$ & 44 & - \\
\hline $\mathrm{H}^{13} \mathrm{CO}^{+}$ & $4-3$ & $346998.338(89)$ & 8.8 & 0.35 & $1.2(-11)$ & 0.46 & - \\
\hline \multirow[t]{2}{*}{$\mathrm{DCO}^{+}$} & $4-3$ & $288143.858(7)$ & 8.8 & 0.35 & $2.1(-11)$ & 1.2 & - \\
\hline & $5-4$ & $360169.778(7)$ & & & & 0.25 & - \\
\hline \multirow[t]{2}{*}{$\mathrm{N}_{2} \mathrm{H}^{+}$} & $3-2$ & $279511.858(11)$ & 7.3 & 0.50 & $2.2(-10)$ & 15 & 16 \\
\hline & $4-3$ & $372672.560(13)$ & & & & 3.4 & 3.6 \\
\hline $\mathrm{N}_{2} \mathrm{D}^{+}$ & $4-3$ & $308422.322(10)$ & 7.3 & 0.50 & $2.5(-11)$ & 0.49 & 0.51 \\
\hline $\mathrm{o}-\mathrm{H}_{2} \mathrm{D}^{+}$ & $1_{10}-1_{11}$ & $372421.385(10)$ & 8 & 0.50 & $4.3(-11)$ & 0.19 & - \\
\hline
\end{tabular}

(1) from the CDMS catalog as of Feb. 2006 (see Müller et al. 2005).

The uncertainties in parenthesis are given in units of the last digit. (2) $X(p)$ means $X \times 10^{p}$.

\section{Implications: evolutionary state of Cha-MMS1}

The deuterium fractionations of $\mathrm{HCO}^{+}$and $\mathrm{N}_{2} \mathrm{H}^{+}$derived in Sect. 3.3 are 3-4 orders of magnitude larger than the cosmic ratio $\frac{[\mathrm{D}]}{[\mathrm{H}]} \sim 10^{-5}$. This enhanced molecular deuteration is typical for low-mass dense cores (e.g. Williams et al. 1998; Crapsi et al. 2005; Parise et al. 2006) and correlates well with CO depletion in prestellar cores (Bacmann et al. 2003). It is understood as a result of $\mathrm{CO}$ freeze out onto the grain surface, $\mathrm{CO}$ being the major destroyer of $\mathrm{H}_{2} \mathrm{D}^{+}$, the key ion in the molecular deuterium chemistry (e.g. Caselli et al. 2003). To understand the different deuteration degrees measured for $\mathrm{HCO}^{+}$and $\mathrm{N}_{2} \mathrm{H}^{+}$in Cha-MMS1, we compare our results with the predictions of chemical models. Roueff et al. (2005) calculated the steady state of models of gasphase chemistry at $10 \mathrm{~K}$. Their three models, Model 1, 2, and 3, have densities of $10^{4}, 10^{5}$, and $10^{6} \mathrm{~cm}^{-3}$, and $\mathrm{C}$ and $\mathrm{O}$ depletion factors of 1,5 , and 15 , respectively. At $10 \mathrm{~K}$, the ortho-to-para ratio of $\mathrm{H}_{2} \mathrm{D}^{+}$is close to 1 (Gerlich et al. 2002), so we deduce a total $\mathrm{H}_{2} \mathrm{D}^{+}$abundance of $\sim 9 \times 10^{-11}$, in agreement with Model 3 and close to the value derived for the evolved prestellar core L1544 (van der Tak 2006). For $\mathrm{N}_{2} \mathrm{H}^{+}$, both the abundance and the deuterium fractionation in Table 2 agree well with the predictions of Model 3, while they are close to Model 2 for $\mathrm{HCO}^{+}$. The degree of deuterium fractionation in $\mathrm{HCO}^{+}$traces therefore lower densities than the $\mathrm{N}_{2} \mathrm{H}^{+}$one. This can be understood if $\mathrm{HCO}^{+}$is depleted at high densities, which is expected in dense cores since $\mathrm{HCO}^{+}$is chemically related to $\mathrm{CO}$. To test this scenario, we computed a model for $\mathrm{HCO}^{+}$and isotopologues with a hole of radius $1800 \mathrm{AU}$ and abundances 2.5 times larger: the fit to the observed spectra was as good as for the model shown in Fig. 2, suggesting that $\mathrm{HCO}^{+}$may indeed be depleted in the inner region. Crapsi et al. (2005) derived a $\mathrm{N}_{2} \mathrm{H}^{+}$deuterium fractionation of $16-23 \%$ for L1544 and 5-10\% for L1521F, which is now known as a Class 0 protostar since its detection by the Spitzer Space Telescope (SST) in the near infrared (e.g. Terebey et al. 2005). Our value for Cha-MMS1 lies in between, which suggests an evolutionary state between L1544 and L1521F if the $\frac{\left[\mathrm{N}_{2} \mathrm{D}^{+}\right]}{\left[\mathrm{N}_{2} \mathrm{H}^{+}\right]}$ratio can be used as a chemical clock.

The higher spatial resolution provided by APEX and the higher densities traced by $\mathrm{CO}(3-2)$ allowed us to resolve the blueshifted wing emission seen earlier in $\mathrm{CO}(1-0)$ (Mattila et al. 1989; Prusti et al. 1991) into two outflows - the second one 
being tentative -, and assign their driving sources. The main outflow is not powered by Cha-MMS1, as Reipurth et al. (1996) suggested, but by the Class I protostar Ced 110 IRS 4. This is consistent with the non-detection of Cha-MMS1 in centimeter continuum emission (Lehtinen et al. 2003). Since we did not find evidence for an outflow driven by Cha-MMS1 itself, the dense core must be in an earlier evolutionary state than the typical young Class 0 protostar IRAM 04191 which does have a powerful outflow (André et al. 1999). However, using the SST archive (http://ssc.spitzer.caltech.edu/), we discovered a source detected at 24,70 and $160 \mu \mathrm{m}$ by the instrument MIPS (PID:37, REQID:3962112), offset from Cha-MMS1 by 5$7^{\prime \prime}$ toward the East. Given the large beams of SEST (22") and SST $\left(6-18^{\prime \prime}\right)$ and their pointing rms accuracies ( $3^{\prime \prime}$ and $1.4^{\prime \prime}$, respectively), we find it conceivable that this mid-infrared source is embedded in the Cha-MMS1 dense core. We measure fluxes of $2.5 \pm 0.8$ and $200 \pm 100 \mathrm{mJy}$ at 24 and $70 \mu \mathrm{m}$. The $24 \mu \mathrm{m}$ flux is $\sim 7$ and 10 times smaller than the fluxes we measured for IRAM 04191 and L1521F using the SST data products of the C2D legacy program (Evans et al. 2003), and the $70 \mu \mathrm{m}$ flux $\sim 4$ and 3 times smaller ${ }^{3}$. In addition, Cha-MMS1 was not detected at $8 \mu \mathrm{m}$ by the instrument IRAC (SST public data, PID:37, REQID:3960320), at a $3 \sigma$ level $\sim 9$ and 7 times lower than the peak fluxes of IRAM 04191 and L1521F. The detection at $24 \mu \mathrm{m}$ implies that Cha-MMS1 already contains a compact hydrostatic object (e.g. Masunaga \& Inutsuka 2000), but the weakness and "redder" color of the 24 and $70 \mu \mathrm{m}$ fluxes and the non-detection at $8 \mu \mathrm{m}$ suggest that it is less evolved than IRAM 04191 and L1521F (e.g. Young \& Evans 2005), since they are at the same distance. Depending on its inclination along the line of sight (see Boss \& Yorke 1995; Whitney et al. 2003, for its effects on the mid-infrared fluxes), it could be at the stage of the first hydrostatic core, i.e. at the very end of the prestellar phase, or have already formed an extremely young Class 0 protostar that has not yet powered any outflow. At a stage intermediate between L1544 and L1521F, Cha-MMS1 would then be the first object found so close to the very beginning of the Class 0 accretion phase.

Acknowledgements. We thank P. André and J. Kauffmann for enlightening discussions about Cha-MMS1 and the SST, and the APEX staff for their help during the observations. BP is grateful to the A. von Humboldt Foundation for a Humboldt Research Fellowship.

\section{References}

André, P., Motte, F., \& Bacmann, A. 1999, ApJ, 513, L57

Bacmann, A., André, P., Puget, J.-L., Abergel, A., Bontemps, S., \& WardThompson, D. 2000, A\&A, 361, 555

Bacmann, A., Lefloch, B., Ceccarelli, C., Steinacker, J., Castets, A., \& Loinard, L. 2003, ApJ, 585, L55

Belloche, A., André, P., Despois, D., \& Blinder, S. 2002, A\&A, 393, 927

Boss, A. P., \& Yorke, H. W. 1995, ApJ, 439, L55

Cabrit, S., \& Bertout, C. 1990, ApJ, 348, 530

Caselli, P., van der Tak, F. F. S., Ceccarelli, C., \& Bacmann, A. 2003, A\&A, 403, L37

Crapsi, A., Caselli, P., Walmsley, C. M., Myers, P. C., Tafalla, M., Lee, C. W., \& Bourke, T. L. 2005, ApJ, 619, 379

Evans, N. J., II, Allen, L. E., Blake, G. A., et al. 2003, PASP, 115, 965

Flower, D. R. 1999, MNRAS, 305, 651

Froebrich, D. 2005, ApJS, 156, 169

Gerlich, D., Herbst, E., \& Roueff, E. 2002, Planet. Space Sci., 50, 1275

Haikala, L. K., Harju, J., Mattila, K., \& Toriseva, M. 2005, A\&A, 431, 149

Hogerheijde, M.R., \& van der Tak, F.F.S. 2000, A\&A, 362, 697

Knude, J., \& Høg, E. 1998, A\&A, 338, 897

Lehtinen, K., Haikala, L. K., Mattila, K., \& Lemke, D. 2001, A\&A, 367, 311

Lehtinen, K., Harju, J., Kontinen, S., \& Higdon, J. L. 2003, A\&A, 401, 1017

Mattila, K., Liljestrom, T., \& Toriseva, M. 1989, in Low Mass Star Formation and Pre-main Sequence Objects, Ed. B. Reipurth, ESO Conf. Workshop Proc., 33, 153

Masunaga, H., \& Inutsuka, S.-I. 2000, ApJ, 531, 350

Müller, H. S. P., Schlöder, F., Stutzki, J., \& Winnewisser, G. 2005, J. Mol. Struct., 742,215

Parise, B., Ceccarelli, C., Tielens, A.G.G.M., Castets, A., Caux, E., Lefloch, B., \& Maret, S. 2006, A\&A, in press

Persi, P., Marenzi, A. R., Gómez, M., \& Olofsson, G. 2001, A\&A, 376, 907

Prusti, T., Clark, F. O., Whittet, D. C. B., Laureijs, R. J., \& Zhang, C. Y. 1991, MNRAS, 251, 303

Redman, M. P., Keto, E., Rawlings, J. M. C., \& Williams, D. A. 2004, MNRAS, 352,1365

Reipurth, B., Nyman, L.-Å., \& Chini, R. 1996, A\&A, 314, 258

Roueff, E., Lis, D. C., van der Tak, F. F. S., Gerin, M., \& Goldsmith, P. F. 2005, A\&A, 438, 585

Terebey, S., Sullivan, A., Fich, M., \& Padgett, D. L. 2005, AAS Meet. Abstr., 207, 165.01

Van der Tak, F. F. S., van Dishoeck, E. F., Evans, N. J., Bakker, E. J., \& Blake, G. A. 1999, ApJ, 522, 991

Van der Tak, F. F. S. 2006, Phil. Trans. R. Soc. Lond. A, in press [astro-ph/0602340]

Ward-Thompson, D., André, P., Crutcher, R., et al. 2006, in Protostars and planets V, Ed. B. Reipurth, D. Jewitt \& K. Keil (Tucson: Univ. Arizona Press), in press

Whitney, B. A., Wood, K., Bjorkman, J. E., \& Cohen, M. 2003, ApJ, 598, 1079

Williams, J. P., Bergin, E. A., Caselli, P., Myers, P. C., \& Plume, R. 1998, ApJ, 503,689

Young, C. H., \& Evans, N. J. 2005, ApJ, 627, 293

Zinnecker, H., Krabbe, A., McCaughrean, M. J., et al. 1999, A\&A, 352, L73

\footnotetext{
${ }^{3}$ Note that the $70 \mu \mathrm{m}$ flux is one order of magnitude weaker than the questionable $3.7 \sigma$ detection of Lehtinen et al. (2001) with ISOPHOT.
} 